\title{
Clinical presentation of a horse-derived biomaterial and its Biocompatibility: A Clinical Case Report
}

\author{
Ki-Tae Koo, Jang-Yeol Park, Jun-Seok Park, Tae-Il Kim, Yang-Jo Seol, Yong-Moo Lee, Young Ku, \\ In-Chul Rhyu, Chong-Pyoung Chung* \\ Department of Periodontology and Dental Research Institute, School of dentistry, Seoul National University, Seoul, Korea
}

\begin{abstract}
Purpose: The objective of this clinical presentation was to present a clinical case series report of socket preservation, sinus augmentation, and bone grafting using a horse-derived biomaterial.

Methods: A horse-derived biomaterial was used in 8 patients for different indications including socket preservation following tooth extraction, osseous bone grafting, and sinus augementation procedures. Surgeries were performed by a well trained specialist and clinical radiographs were obtained at designated intervals. Biopsy cores of $2 \times 8 \mathrm{~mm}$ prior to implant placement was obtained following a healing interval of 4-6 months. A clinical and histologic evaluation was performed to evaluate the clinical effectiveness and biocompatibility of the biomaterial.

Results: All surgeries in 8 patients were successful with uneventful healing except for one case with membrane exposure that eventually resulted with a positive outcome. Radiographic display of the healing phase during different intervals showed increased radiopacity of granular nature as the healing time increased. No signs of adverse effect or infection was observed clinically and the tissues surrounding the biomaterial seemed well-tolerated with good intentional healing. The augmented sinuses healed uneventfully suggesting in part, good biocompatibility of the biomaterial. Dental implants placed following socket preservation were inserted with high initial torque suggesting good initial stability and bone quality.

Conclusions: Our results show that at least on a tentative level, a horse-derived biomaterial may be used clinically in socket preservation, sinus augmentation, bone grafting techniques with good intentional healing and positive results.
\end{abstract}

(J Korean Acad Periodontol 2009;39:287-291)

KEY WORDS: biocompatible materials ; bone substitutes ; wound healing.

\section{INTRODUCTION}

The shift in therapeutic concepts from resection to regeneration has significantly changed the practice of periodontologly in recent years. Under the assumption that proper use and manipulation of bone replacement grafts can alter the biologic response from a regenerative to a reparative pattern, a robust interest and demand for bone replacement grafts with forementioned biologic potential have grown significantly. Also, the introduction and acceptance of dental implants as a predictable treatment modality has created new indications

Correspondence: Dr. Chong-Pyoung Chung

Department of Periodontology, School of Dentistry, Seoul National University, 28 Yongon-Dong, Chongno-Ku, Seoul 110-749, Korea.

E-mail: ccpperio@snu.ac.kr,, Tel: 82-2-2072-2289, Fax: 82-2-744-0051. Received: Jul 21, 2009; Accepted: Jul 29, 2009 for the use of bone replacement grafts eventually resulting in increased demand and search for products with higher quality and superior biologic compatibility.

Various graft materials including autogenous, allogeneic, xenogeneic and alloplastic bone graft materials have been used in preserving the alveolar ridge following tooth extraction ${ }^{1)}$. Autogenous bone, still considered the gold standard in bone grafting has been used with some success. Albeit the excellent biologic properties, limitations exist in availability, patient morbidity, increased chair time and irregular resorption rate. Allogeneic bone obtained from a different human source provides an alternative to autogenous bone, but the fear of disease transmission persists despite evidence to the contrary. This concern has driven the market to produce biocompatible alter- 


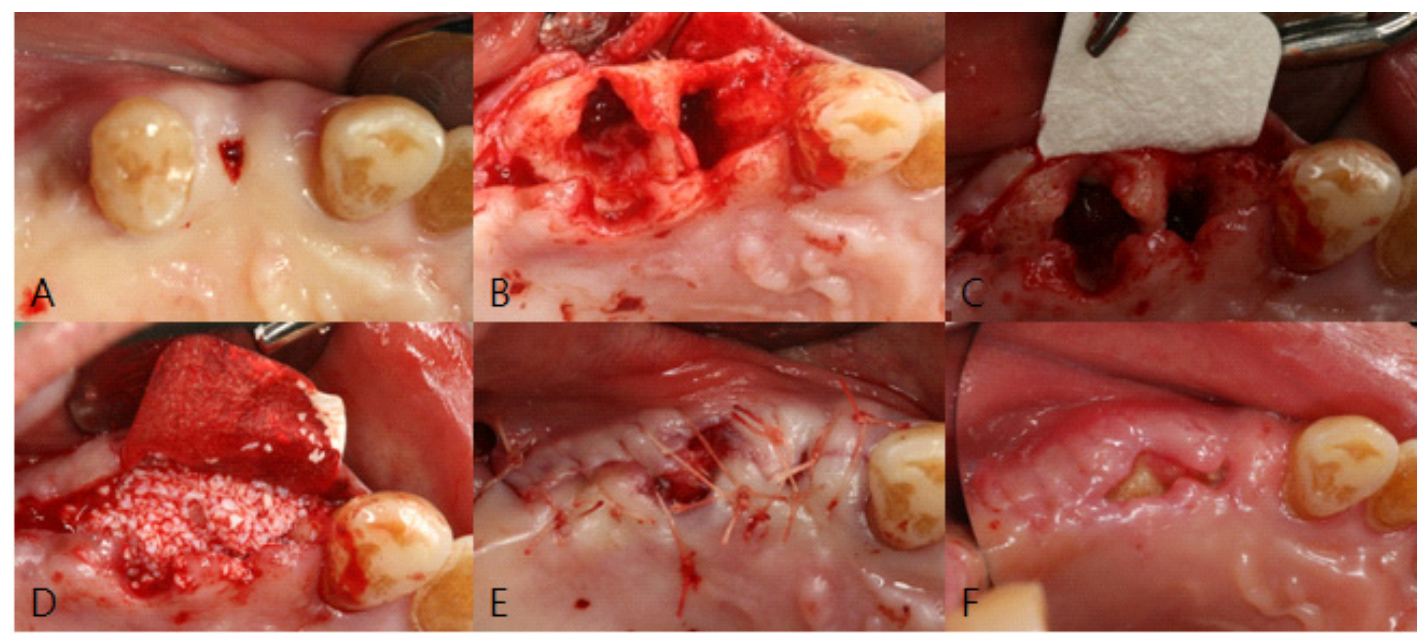

Figure 1. Clinical illustration of the maxillary right premolar region grafted with a horse-derived bone substitute and a collagen membrane following extraction of the second premolar. A.clinical photograph prior to extraction $(\times 2)$ B. extraction socket following extraction of the second premolar $(\times 2)$ C.adaptaion of the collagen membrane to the extraction site $(\times 2)$ D.clinical photograph following the graft procedure ( $\times 2)$ E.clincal photograph after suturing F.clinical photograph following suture removal at 1 week $(\times 2)$.

natives to allogeneic bone.

In particular, a deprotenized bovine bone mineral has been extensively studied and is currently well accepted clinically by the majority of users involved in clinical dentistry ${ }^{2,3)}$. However, despite heat treatment and prophylactic measures during processing to prevent disease transmission, safety concerns still remain especially with the discovery of bovine spongiform encephalopathy ${ }^{4}$. The controversy regarding this epidemic event remains unsolved though many attempts have been made to draw a definitive conclusion. A need for a complete disease transmission free donor has propagated research and development of a bone replacement graft from sources other than bovine origin. The objective of this clinical report was to observe both clinically and histologically, the biocompatibility and physical properties of a horse-derived bone biomaterial.

\section{CLINICAL CASES}

A horse-derived biomaterial was used in 8 patients admitted to Seoul National University, Department of Periodontology for different indications including socket preservation following tooth extraction, osseous bone grafting, and sinus augmentation procedure.
Surgeries were performed by a well trained specialist. Clinical radiographs were obtained at different intervals while core biopsies of $2 \times 8 \mathrm{~mm}$ were acquired prior to implant placement. This protocol was approved by the Institutional Review Board at Seoul National University Dental Hospital.(CDE 09002)

\section{Case 1}

Socket preservation technique was applied to the maxillary right pre-molar region following extraction of the second premolar diagnosed as having a hopeless prognosis. The first premolar was extracted beforehand. Following extraction and meticulous debridement of the site, a horse-derived bone substitute (OCS-H, NIBEC, Seoul, Korea) combined with a resorbable collagen membrane was used. Due to the insufficient volume of soft tissue, primary closure was not achieved. Despite the open dehiscence of the wound, infection of the wound area was avoidable and the surgical site healed uneventfully with secondary intentional healing (Fig. 1). Note the increased radiopacity of granular nature at 6 months healing (Fig. 2). Also, excellent ridge contour maintenance was observed both clinically and radiographically. Histologic observations via biopsy 
Figure 2.

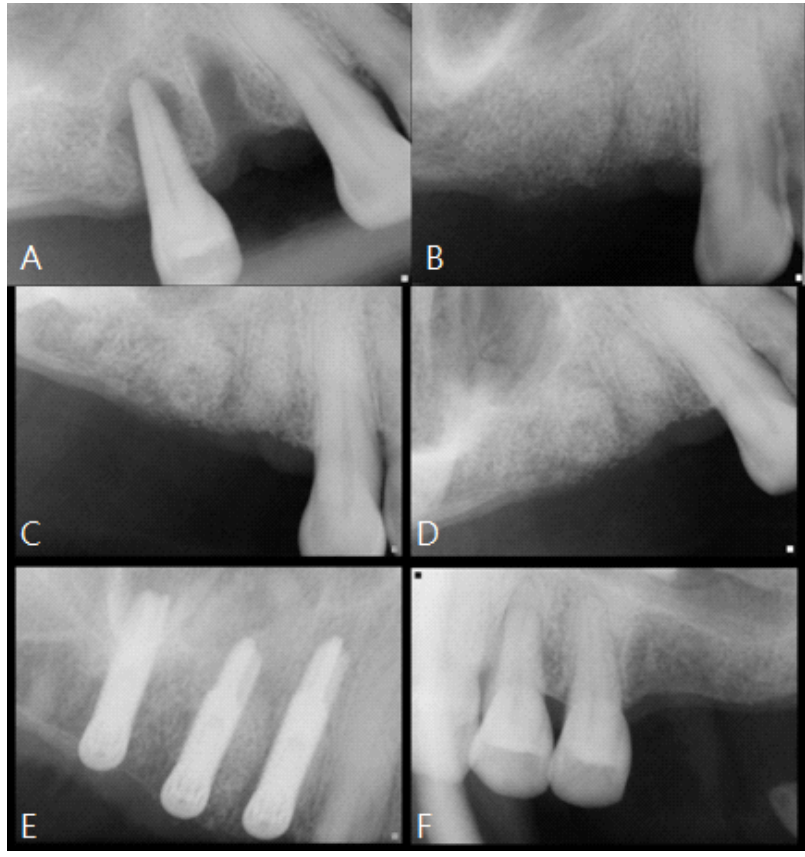

Figure 3.

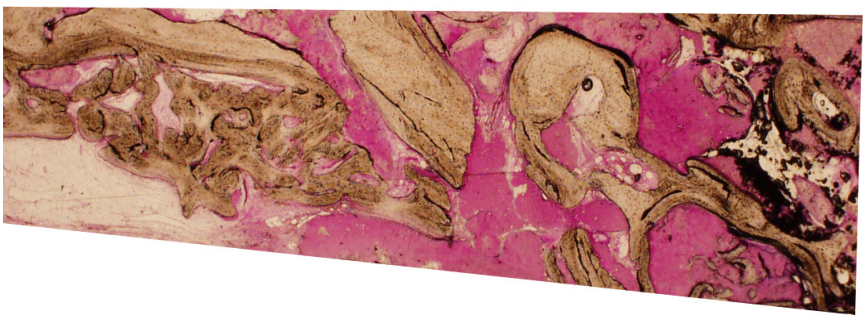

Figure 2. Radiographs illustrating the grafted extraction socket site from pre-extraction to implant placement at 6 months. A maxillary right second premolar before extraction B.radiographic representation of the site grafted with OCS-H at 1 month post-surgery C.healing at 3 months post-surgery D.healing at 6 months post-surgery E. 3 dental implants were placed successfully in the extraction socket site grafted with OCS-H after 6 months F. contralateral side also grafted with OCS $-\mathrm{H}$ following tooth extraction.

Figure 3. Photomicrograph of a $2 \times 8 \mathrm{~mm}$ biopsy core obtained prior to implant placement. (basic fuchsin \& methylene blue staining, Original magnification $\times 20)$.
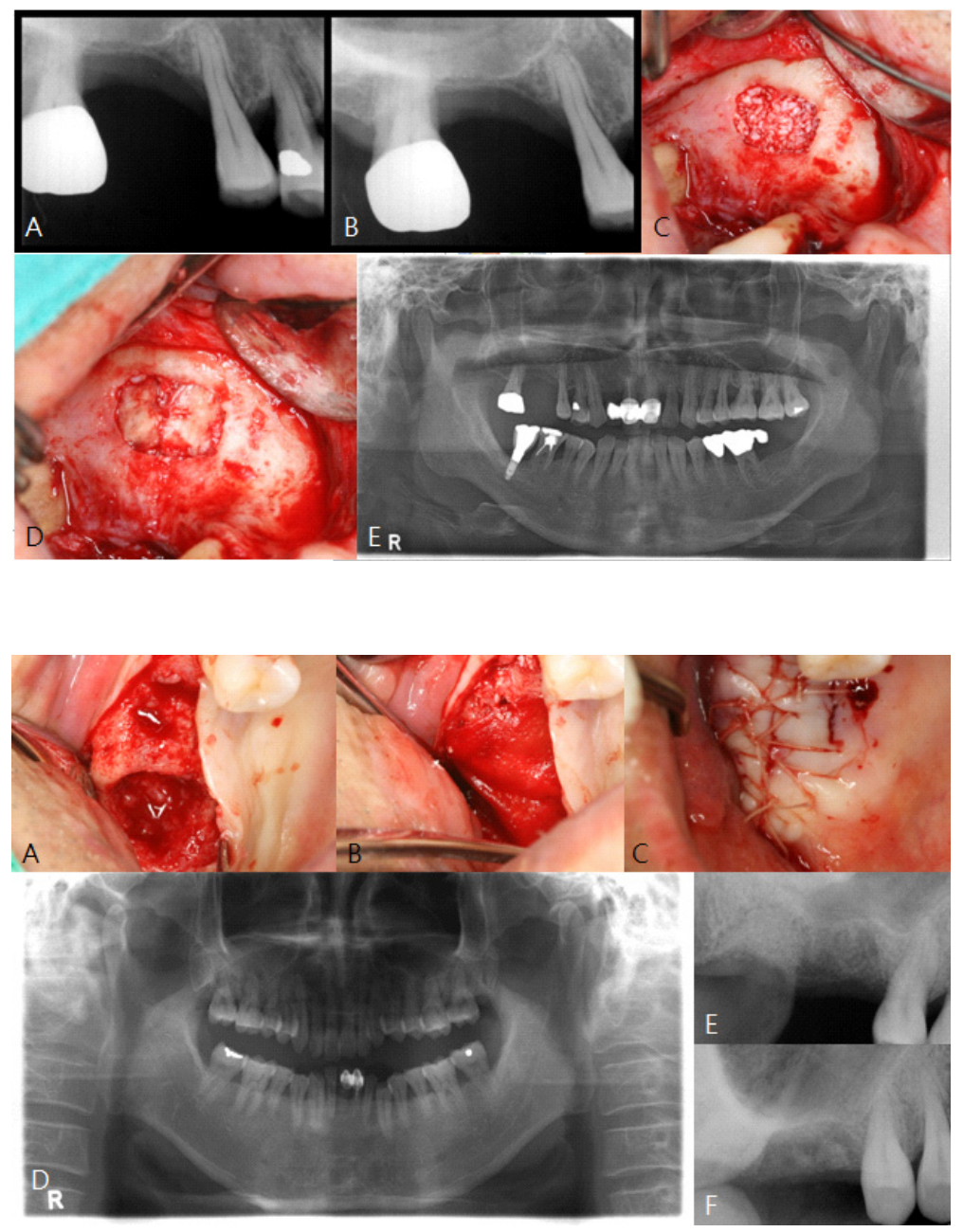

Figure 4 . Clinical photograph and radiograph illustrating a severely pneumatized maxillary sinus grafted with OCS-H.

Figure 5. Clinical and radiographic presentation grafting procedure with a horse-derived bone substitute on the maxillary second molar region. A clinical photograph of the region prior to grafting $(\times 2)$ B. a membrane was secured using the buccal and palatal flaps $(\times 2)$ C.an attempt was made to achieve good primary intentional healing $(\times 2)$ D.panoramic view of the right maxillary second molar region prior to extraction E.radiograph taken at 2 weeks post-op F.radioghic presentation of the grafted area at 6 months. 
cores obtained prior to implant placement revealed new bone formation throughout the grafted defect. Most of the specimens showed new bone formation extending to the coronal part of the defect with even deposition around the graft material. The appearance of the newly formed bone exhibited trabecular patterns of mature bone with smooth consistency and continuity with the graft material suggesting a well-tolerated biocompatibility of the biomaterial.

\section{Case 2}

A sinus augmentation with a horse-derived bone substitute (OCS-H, NIBEC, Seoul, Korea) using the lateral approach was performed on maxillary right first molar area with severe pneumatization (Fig. 4). Residual bone of less than $2 \mathrm{~mm}$ was successfully grafted with a horse-derived bone substitute using the lateral-window approach with no signs of infection and uneventful healing. Increased radiopacity below the sinus floor was observed following a healing period of 6 months. A dental implant was successfully placed at this time period.

\section{Case 3}

A decision was made to intervene and graft the maxillary second molar area due to delayed and nonspontaneous healing. (Fig 5) After removal of granulation and fibrous tissue, a horse-derived bone substitute (OCS-H, NIBEC, Seoul, Korea) and a resorbable collagen membrane was used. Attempts were made to achieve good primary closure and uneventful healing was observed throughout the 6 month healing period. Note the increased density and maturation of bone at the 6 month interval as depicted in the follow-up radiographs.

\section{DISCUSSION}

Eight patients with different indications were subjected to various procedures that involved the use of a horse derived bone substitue. Throughout the observation period of the successive surgeries that were performed, no signs of adverse effect or infection was obeserved both clinically and radiographically. Uneventful healing was observed in most of the cases except for one case with membrane exposure that eventually resulted with a positive outcome. Radiographic anlysis of the surgical sites showed increased radiopacity of granular nature as the healing time increased. Clinically, the biomaterial seemed well-tolerated with good intentional healing and harmony with the native surrounding tissues. Excellent ridge contour maintenance was observed both clinically and radiographically. The augmented sinuses healed uneventfully suggesting in part, good biocompatibility of the biomaterial. Dental implants placed following socket preservation were inserted with high initial torque suggesting good initial stability and bone quality. Histologic observations at 4-6 months healing intervals revealed new bone formation throughout the grafted defect. New bone formation extending to the coronal part of the defect showed appearance of mature bone with even continuity with the graft material. Also, appearance of full integration between the grafted bone and newly formed bone was observed.

Our results show that at least on a tentative level, a horse-derived biomaterial may be used clinically in socket preservation, sinus augmentation, bone grafting techniques with good intentional healing and positive results.

\section{REFERENCES}

1. Jensen SS, Aaboe M, Pinholt EM. Tissue reaction and material characteristics of four bone substitutes. Int $\mathrm{J}$ oral Maxillofacial Implant 1966;11:55-66.

2. Artzi Z, Tal H, Dayan D. Porous bovine bone mineral in healing of human extraction sockets:Histochemical observations at 9 months J Periodontol 2001;72:152-159.

3. Carmagnola D, Adriaens P, Berglundh. Healing of human 
extraction sockets filled with Bio-oss. Clin Oral Impl Res 2003;14:137-143.

4. Wenz B, Oesch B, Horst M. Analysis of the risk of transmitting bovine spongiform encephalopathy through bone grafts derived from bovine bone. Biomaterials 2001;22: 1599-1606.

5. Yukna RA, Mayer ET, Brite DV. Longitudinal evaluation of durapatite ceramic as an alloplastic implant in periodontal defects after 3 years. J Periodontol 1984;55:633-637.

6. Meffert RM, Thomas JR, Hamilton KM, Brownstein CN. Hydroxyapatite as an alloplastic graft in the treatment of human periodontalosseous defects. J Periodontol 1985;56:63-73.

7. Klinglsberg J. Scleral allograft in the repair of periodontal osseous defects. NY state Dent J 1972;38:418-420.

8. Radents WH, Collings CK. The implantium of plaster of paris in the alveolar process of the dog. J Periodontol 1965;36:357-364.

9. Stephan T, Sonis RC, Williams, Marjorie K, Jeffcoat. Healing of spontaneous periodontal defects in dogs treated with xenogenic demineralized bone. J Periodontol 1979;50: 23-27.

10. Pinholt EM, Bang G, Haanaes HR. Alveolar ridge augmentation in rats by Bio-oss. Scand J Dent Res 1991;99:154-161.

11. Klinge $\mathrm{B}$, Alberius $\mathrm{P}$, Isaksson $\mathrm{S}$, Jonsson AJ. Osseous response to implanted natural bone mineral and synthetic hydroxylapatite ceramic in the repair of experimental skull bone defects. J oral maxillofac Surg 1992;50:241-249.

12. Young C, Sandstedt P, Skoglund A. A comparative study of anoganic xenogenic bone and autogenous bone implants for bone regeneration in rabbits. Int $\mathrm{J}$ oral Maxillofacial Implant 1999;14:72-76.

13. Skoglund A, Hising P, Young C. A Clinical and histologic examination in humans of the osseous response to implanted natural bone mineral. Int $\mathrm{J}$ oral Maxillofacial Implant 1997;12:194-199.

14. Becker W, Clokie C, Sennerby L, Urist MR, Becker BE. Histologic findings after implantation and evaluation of different grafting materials and titanium micro screws into extraction sockets: case reports. J Periodontol 1998;68:414-421.

15. Goldberg VM, Stevenson S. Natural history of autografts and allografts. Clin Orthop Related Res 1987;225:7-15.

16. Burchardt H. The biology of bone graft repair. Clin Orthop 1983;174:28-32.

17. Brown P, Preece MA, Will RG. "Friendly fire" in medicine: hormones, homografts, and Creutzfeldt-Jakob disease in the UK. Lancet 1992;340:24-27.

18. Shin-Il Yeo, Sung-Hwan Park, Woo-chang Noh et al. A comparative analysis of basic characteristics of several deproteinized bovine bone substitutes. J Korean Acad Periodontol 2009;39:149-156.

19. Woo-Kyung Son, Seung-Yun Shin, Seung-Min Yang, Seoung-Beom Kye. Maxillary sinus floor augmentation with anorganic bovine bone: Histologic evaluation in humans. J Korean Acad Periodontol 2009;39:95-102.

20. Artzi Z, Tal H, Dayan D. Porous bovine bone mineral in healing of human extraction sockets. Part I : histomorphometric evaluations at 9 months. J Periodontol 2000;71: 1015-1023. 


\section{Erratum}

The article type of "Patient compliance with supportive periodontal therapy" ( $\mathrm{J}$ Korean Acad Periodontol 2009;39;193-198) is not a clinical case, but an original article. 